\title{
MedienPädagogik
}

Zeitschrift für Theorie und Praxis der Medienbildung

Themenheft Nr. 34:

Forschung und Open Educational Resources - Eine Momentaufnahme für Europa Herausgegeben von Markus Deimann

\section{Systematic Literature Review zu Open Educational Practices (OEP) in der Hochschule im europäischen Forschungskontext}

Franziska Bellinger und Kerstin Mayrberger

\begin{abstract}
Zusammenfassung
Open Educational Practices (OEP) erscheinen in der Debatte um Openness im Bildungskontext und in Verbindung mit Open Educational Resources (OER) trotz einer nachweislichen Verbindung als noch relativ unterrepräsentiert. So wird hier der Annahme gefolgt, dass es aus theoretischer, empirischer wie diskursiver Perspektive notwendig erscheint für eine Kultur des Teilens und weiter eine offene Bildung im Sinne von Open Education und Open Pedagogy, die sich in Variationen von OEP manifestieren, zu sensibilisieren. Auf Grund der Bedeutung von OEP für den derzeitigen Transformationsprozess im Hochschulkontext unter den Bedingungen der Digitalisierung liefert der Beitrag einen systematischen Überblick zu Forschungsarbeiten zu OEP. Dabei findet eine Orientierung entlang der übergreifenden Fragestellung, in welchen Zusammenhängen und unter welchen Fragestellungen OEP im Kontext Hochschule erforscht werden und mit welchem Begriffsverständnis gearbeitet wird, statt. Das Ergebnis des Literature Reviews zeigt, dass in den Forschungsarbeiten ein variantenreiches Verständnis von OEP thematisiert wird. In Abhängigkeit dessen wie das Verhältnis der Praktiken der Subjekte und die Praxis jeweils gefasst werden, lassen sich vier Dimensionen einer OEP im Kontext von Openness identifizieren. Der Beitrag plädiert daher für eine begriffliche Schärfung von OEP im jeweils spezifischen Verwendungszusammenhang. Die Autorinnen forcieren dabei eine praxistheoretische Perspektive. Es wird argumentiert, dass ein praxistheoretischer Zugang es ermöglicht, offene Bildungspraktiken für den Hochschulkontext theoretisch zu fundieren und empirisch zu untersuchen, um damit einen Beitrag zur zeitgemässen Mediendidaktik respektive Medienpädagogik leisten zu können.
\end{abstract}

Systematic Literature Review on Open Educational Practices (OEP) in Higher Education in the European research area

\footnotetext{
Abstract

Despite a proven link Open Educational Practices (OEP) appear to be still relatively underrepresented in relation to Open Educational Resources (OER) in the debate on Openness in the educational context. Thus, it follows the assumption that it seems
} 
necessary from a theoretical, empirical and discursive perspective for a culture of sharing and to further sensitise an open education in the sense of Open Pedagogy, which manifests itself in variations of OEP. Due to the importance of OEP for the current transformation process in the context of higher education under the conditions of digitisation, the article provides a systematic overview of research on OEP. Thereby, an orientation takes place along the overreaching question in which contexts and with which questions OEP are researched in higher education and which understanding is hereby inherent. The result of the Literature Review shows that the research work addresses a varied understanding of OEP. Four dimensions of an OEP can be identified in the context of Openness, depending on how the relationship between the practices of the subjects and the practice is taken in each case. The contribution therefore advocates a conceptual sharpening of OEP in the specific context of use. The authors force a practical theoretical perspective. It is argued, that a practical theoretical approach enables to theoretically substantiate and empirically investigate Open Educational Practices for the context of higher education, in order to make a contribution to contemporary media didactics and media education.

\section{Sondierung eines expansiven Forschungsfeldes: Eine Einleitung}

In der Diskussion um Offenheit im formalen Bildungskontext fällt zumeist vorweg das Schlagwort Open Educational Resources (OER). An zweiter Stelle findet nicht selten eine Kontextualisierung um Digitalisierung statt, sodass damit das nächste Schlagwort, die sogenannte «Digitale Bildung», in die Diskussion - wie sie derzeit vor allem bildungs-, sozial- und auch wirtschaftspolitisch vorangetrieben wird - hinzugezogen wird. Weniger selbstverständlich wird der pädagogische und didaktische Kontext benannt, wenn es um Openness und Bildung geht sowie die entsprechenden Open Educational Practices (OEP).

Die aktuellen Debatten um Offenheit im Bildungskontext sind durch ihre ebenfalls politische Relevanz normativ aufgeladen und werden entlang Idealen geführt bis hin zu ideologischen Tendenzen, wenn es um die «richtigen» Begriffe und entsprechenden Open Practices geht. Daher lässt sich der vorherrschende Diskurs auch als Bewegung im Kontext Openness charakterisieren, wie bereits die «Capetown Open Education Declaration» 2007 konstatierte ${ }^{1}$. Open Education spielt auf vielen, gleichermassen relevanten, Ebenen eine Rolle: Sei es - um einige exemplarisch zu nennen - aus bildungspolitischer Sicht, wenn es darum geht durch offene Zugänge chancengerechte sowie qualitativ hochwertige Bildung zu ermöglichen, wie es die UNESCO forciert (Peter 2018). Aus sozialpolitischer Richtung, um die Kosten für Bildungsmaterialien und Lehrbücher zu senken, wie es die Open-Textbook-Debatte in den USA und UK kennzeichnet ${ }^{2}$, oder wenn für staatlich finanzierte Forschung und

1 http://www.capetowndeclaration.org/

2 siehe z.B. https://research.cehd.umn.edu/otn/ 
Entwicklung freie Zugänglichkeit zu den Ergebnissen eingefordert wird (siehe u.a. Förderbedingungen des Bundesministeriums für Bildung und Forschung). Daneben reihen sich auch pragmatische Sichtweisen, sodass mit Hilfe von vielfältigen offenen und freien Bildungsmaterialien durch das Prinzip des Teilens von Ressourcen zur Materialerstellung einfacher auf bereits existierende digitale Bildungsmedien zugegriffen werden kann. Somit können zeitgemässe Lehre oder frei zugängliche OnlineLernangebote an Hochschulen potenziell zügiger adaptiert und realisiert sowie im Anschluss die eigens erstellten OER wiederum zur Verfügung gestellt werden (siehe zahlreiche Beispiele aus dem Kontext der OERinfo-Linie 20183).

Bereits die Capetown Open Education Declaration (2007) setzt mit ihrem Untertitel prominent bei OER an, wenn es zuvorderst heisst «Unlocking the promise of open educational resources». Die ersten beiden Absätze dieser Erklärung beziehen sich sowohl auf digitale Ressourcen als auch auf eine neue bzw. veränderte pädagogische Handlungspraxis, sowie Einstellungen und Haltungen der beteiligten Lehrenden und Lernenden hinsichtlich eines Konsens der gemeinsamen Produktion, Nutzung und damit einer Kultur des Teilens mit partizipativen Zügen. ${ }^{4}$ Neben genannten, vor allem politisch und idealistisch motivierten, (Beweg-)Gründen bleibt also aus fachlicher, sprich medienpädagogischer und besonders mediendidaktischer, Perspektive zu fragen, wie der Ansatz einer OEP einzuordnen ist in Abstimmung mit einem jeweiligen Verständnis von OEP als Praktik oder Praxis und inwiefern hier eine Weiterentwicklung oder Ergänzung bestehender (reform-)pädagogischer Ideen und Konzept erfolgt oder gar unter den Vorzeichen von Offenheit und unter dem Dach einer Open Education aus (medien-)pädagogischer und mediendidaktischer Perspektive etwas Neues im Entstehen ist. So schlägt bspw. Naidu (2016) vor OEP eine breitere und umfassendere Bedeutung zukommen zu lassen, und Koseoglu und Bozkurt (2018) sprechen in diesem Zusammenhang von OEP als umfassendes Rahmenkonzept: «useful umbrella term to bring all the different dimensions of openness in education under one roof, with a focus on the processes of education» (ebd., 453).

3 Vgl. BMBF geförderte OERinfo-Linie sowie die Dokumentation der OERinfo-Linie als Sonderband des Fachmagazins Synergie (https://www.synergie.uni-hamburg.de/publikationen/sonderbaende/oer-info-2017-2018.html).

4 Siehe dazu die ersten beiden Absätze im Originalwortlaut: «We are on the cusp of a global revolution in teaching and learning. Educators worldwide are developing a vast pool of educational resources on the Internet, open and free for all to use. These educators are creating a world where each and every person on earth can access and contribute to the sum of all human knowledge. They are also planting the seeds of a new pedagogy where educators and learners create, shape and evolve knowledge together, deepening their skills and understanding as they go. This emerging open education movement combines the established tradition of sharing good ideas with fellow educators and the collaborative, interactive culture of the Internet. It is built on the belief that everyone should have the freedom to use, customize, improve and redistribute educational resources without constraint. Educators, learners and others who share this belief are gathering together as part of a worldwide effort to make education both more accessible and more effective.» (http://www.capetowndeclaration.org/read-the-declaration). Nach 10 Jahren wurde diese Declaration (kurz CPT) nochmals bekräftigt und unter dem Hashtag \#CPT10 um «Ten Directions to Move Open Ecuational Forward» erweitert, um die Bewegung weiter zu fördern (http://www.capetowndeclaration.org/cpt10/). 
An dieser Stelle könnte man ebenso einwenden, dass es möglicherweise auf ein «Henne-Ei-Problem» hinausläuft, inwiefern eine OEP insbesondere von dem Vorhandensein von OER abhängig sei oder es OER ohne eine OEP nicht gäbe. Um diesen Fragen jenseits von politischer oder idealistischer Motivation und Perspektive nachzugehen, wird hier vor erziehungswissenschaftlichem Hintergrund eine forschende mediendidaktische Perspektive eingenommen, die sich systematisch mit Forschungsarbeiten der letzten zehn Jahre zu OEP im europäischen Hochschulkontext auseinandersetzt. Hiermit erfolgt zugleich ein Beitrag zur Forschung im Feld OEP und OER, die im Verhältnis zu praxisbezogenen Entwicklungen und Vernetzungsaktivitäten sowie Projekten, die die Schaffung von Infrastruktur und Awareness ausgehend vom Thema OER forcieren (was sich zumindest für den deutschsprachigen Raum feststellen lässt), unterrepräsentiert ist (vgl. u.a. Mayrberger und Hofhues 2013; Deimann 2018; Bellinger, Bettinger, und Dander 2018). Dass eine forschungsorientierte Perspektive zunehmend an Relevanz gewinnt, zeigen erste internationale Studien und Forschungsansätze (s. ausführlicher Abschnitt «Stand der Forschung: Ein systematisches Review» zum Forschungsstand von OEP im Hochschulkontext). Zugleich stellt Deimann (2018) in seiner Zusammenschau abschliessend fest:

«Eine priorisierte wissenschaftliche Ausrichtung führt dann zur Herausforderung, die vielen mit Open Education verbundenen Projekte, Maßnahmen und Initiativen auf ihre Wirksamkeit hin zu identifizieren und anhand bestimmter, forschungsleitender Fragestellungen zu untersuchen» (Deimann 2018, 72).

Der vorliegende Beitrag möchte nunmehr dazu beitragen, diese Forschungslücken bezogen auf OEP und den Kontext Hochschule zu füllen. So wird in diesem Beitrag entlang der übergreifenden Fragestellung, in welchen Zusammenhängen und unter welchen Fragestellungen OEP im Kontext Hochschule bis dato im europäischen Raum erforscht werden und mit welchem Begriffsverständnis gearbeitet wird, eine systematische Literaturanalyse vorgenommen.

Da die Autorinnen dem Feld nicht frei von Vorwissen begegneten, wird im Sinne des Transparenzmachens der leitenden Vorabannahme für das Literature Review im nachfolgenden Abschnitt 2 ein konzeptioneller Bezugsrahmen hinsichtlich OER und OEP aufgezogen. Im Zuge dessen wird in aller Kürze ebenfalls die hier eingenommene Perspektive auf Digitalisierung und Digitalität skizziert, sodass die nachfolgende Analyse daran anknüpfen und abschliessend darauf rückbezogen werden kann. Der sehr umfassende Abschnitt 3 ist der Abhandlung des methodischen Vorgehens des hier gewählten systematischen Literature Reviews vorbehalten sowie der Darstellung der Ergebnisse und deren Diskussion. Der Beitrag schliesst mit einem Definitionsvorschlag von OEP auf Basis der Analyse und vor dem Hintergrund mediendidaktischer Forschungsbedarfe, der den Praxisbegriff reflektiert und Anknüpfungspunkte für mediendidaktische Forschungsbemühungen im Kontext zu OEP für den formalen Bildungskontext Hochschule bietet. 


\section{OEP im Kontext: Reflexion relevanter Bezugspunkte aus einer mediendidaktische}

\section{Perspektive}

OEP und OER sind konzeptionell verbunden und je nach Beobachtungsperspektive von OEP als Praktik oder Praxis (vgl. Alkemeyer 2017, 145), im vorliegenden Kontext im Zusammenhang mit Lehr- und Lernpraxen oder einer offenen Praktik für den Bildungsbereich Hochschule, zu reflektieren.

So sehr in diesem Beitrag der Fokus auf OEP liegt, so sinnvoll ist es zugleich für das Verständnis der derzeitigen (inter-)nationalen Diskussion damit zu beginnen aufzuzeigen, was im Zuge dieses Beitrags unter OER verstanden wird, weil diese in engen Begriffsverständnissen häufig als Kern von OEP begriffen werden.

Neben dem Bedarf das Verständnis von OEP inhaltlich in ihrer Reichweite zu schärfen, besteht auch ein Bedarf ihn theoretisch für den jeweiligen Kontext zu konkretisieren. Dazu zählt auch eine OEP, die eng an die Verwendung von OER gebunden wird. Im Gegensatz zum breiten oder bisweilen diffusen Verständnis von OEP handelt es sich bei OER immer um etwas, das man konkret verwenden oder mit dem man etwas machen kann. Unter OER fallen, so man der im deutschsprachigen Raum häufig angeführten Definition der UNESCO (2016) folgt:

«Bildungsmaterialien jeglicher Art und in jedem Medium, die unter einer offenen Lizenz stehen. Eine solche Lizenz ermöglicht den kostenlosen Zugang sowie die kostenlose Nutzung, Bearbeitung und Weiterverbreitung durch Andere ohne oder mit geringfügigen Einschränkungen. Dabei bestimmen die Urheber selbst, welche Nutzungsrechte sie einräumen und welche Rechte sie sich vorbehalten. Open Educational Resources können einzelne Materialien, aber auch komplette Kurse oder Bücher umfassen. Jedes Medium kann verwendet werden. Lehrpläne, Kursmaterialien, Lehrbücher, Streaming-Videos, Multimedia-Anwendungen, Podcasts - all diese Ressourcen sind OER, wenn sie unter einer offenen Lizenz veröffentlicht werden». ${ }^{5}$

Allerdings muss an dieser Stelle darauf hingewiesen werden, dass sich bisher keine Definition zu offenen und freien Bildungsmaterialien oder Bildungsmedien eindeutig durchgesetzt hat ${ }^{6}$. Doch wird der Begriff «offen» oder «open» im Zuge von Open Educational Resources (OER) zumeist entlang der $5 R$ activities von David Wiley (2017) definiert:

- «Retain - the right to make, own, and control copies of the content (e.g., download, duplicate, store, and manage)

- Reuse - the right to use the content in a wide range of ways (e.g., in a class, in a study group, on a website, in a video)

5 Siehe https://www.unesco.de/bildung/open-educational-resources.

6 Für einen Überblick zur Entwicklung von OER und der open-Content-Bewegung siehe beispielsweise folgende Zeitleiste die seit 1997 relevante Stationen benennt unter https://openlab.blogs.uni-hamburg.de/ timeline-development-of-oer-in-germany/. 
- Revise - the right to adapt, adjust, modify, or alter the content itself (e.g., translate the content into another language)

- Remix - the right to combine the original or revised content with other material to create something new (e.g., incorporate the content into a mashup)

- Redistribute - the right to share copies of the original content, your revisions, or your remixes with others (e.g., give a copy of the content to a friend)» (Wiley 2017).

Insbesondere die Sichtweise auf die 5R, die als «Recht auf»zu verstehen sind und sich beispielsweise in der Lizensierung der Creative Commons operationalisieren ${ }^{7}$, stellt einen Indikator für die sich streitenden Geister dar. Denn ein fachlich zentraler wie sensibler Punkt in der Diskussion um die OER(-Bewegung) ist die Frage nach der Lizensierung von OER und inwiefern OER alle der genannten Aktivitäten in der UNESCO-Definition auch ermöglichen und damit «echte» OER seien ${ }^{8}$.

Eine solche Diskussion birgt zugleich die Gefahr, dass die Openness-Bewegung zu sehr auf die technologischen als die ebenso relevanten sozialen und kulturellen Implikationen fokussiert oder gar zurückgehalten wird. Um ein konstruktives statt konkurrierendes Verhältnis zwischen einem weiten OEP Verständnis, das auch eine Open Pedagogy einschliesst, und einem Verständnis von OEP, das auf die Anwendung der $5 R$ besteht, herzustellen haben Wiley und Hilton nunmehr den Begriff einer «OER-Enabled Pedagogy» (Wiley und Hilton 2018) eingeführt.

Es konnte gezeigt werden, dass es sich bei der hier vorliegenden Perspektive auf OER in ihrer gesamten Breite möglicher Granularität hin betrachtet um eine Form medialer Artefakte, oder bei einzelnen OER-Materialien um ein mediales Artefakt, handelt, welches (je nach Perspektive auf OEP) den Anker für die darauf bezogene(n) Praktik(en) darstellt. Im Sinne der hier herangezogenen theoretischen Perspektive liesse sich mit Reckwitz (2003) prüfen, inwiefern die folgende auf Massenmedien bezogene Feststellung sich auch heute auf OER beziehen liesse:

«Als Träger medialer Praktiken stellt sich das medienverwendende Subjekt aus praxeologischer Perspektive weder als ein bloßes Objekt medialer Informationsströme dar noch als völlig ungebunden hinsichtlich seiner individuellen Instrumentalisierung von Medien. Vielmehr lässt sich das mediennutzende Subjekt nun als jemand analysieren, dem die Techniken des Mediengebrauchs zu ,Techniken des Selbst' werden, so dass sich durch die medialen Praktiken bestimmte ,innere“ Kompetenzen und Dispositionen aufbauen» (ebd., 286).

7 Für Informationen zu Creative Commons Lizenzierungsmodellen siehe hier https://creativecommons.org/ und hier https://de.wikipedia.org/wiki/Creative_Commons.

8 Wenngleich die hier angeführte OER Definition grundsätzlich auch analoge Bildungsmedien, wie ein Papierbuch oder ein Foto, als OER zu klassifizieren einschlösse, so sie mit entsprechendem Lizenznachweis versehen wären, wird hier der Perspektive gefolgt, vorrangig digitale Materialien als OER zu fassen, da die erweiterte Nutzungsperspektive entlang der $5 \mathrm{R}$ und insbesondere des Re-Mixens mit einer entsprechend dokumentierten (digitalen) Ablage in Form einer Versionierung inklusive Metadaten auf einem entsprechenden Repositorium alltagsnäher und zukunftsorientierter erscheint. 
Darüber hinaus ist auch die Perspektive von Bellinger, Bettinger und Dander (2018) aufschlussreich, die OEP «als Bündel von sozial situierten Tätigkeiten begreifen, welche im Rahmen einer bestimmten Domäne - hier (Offenheit) - an ein spezifisches Set von Normen gekoppelt sind». OEP werden entsprechend nicht als präskriptives bewusstes Handeln eingeordnet, sondern «als Set von vorbewussten, inkorporierten Routinen verstanden, deren Logik(en) sich erst in Performanzen zeigt bzw. zeigen» (ebd., 114). Insofern wird in diesem Beitrag für eine forschungsbezogene Auseinandersetzung mit OEP eine Konkretisierung im Anschluss an Alkemeyer (2017) vorgenommen, der wie folgt zwischen Praxis und Praktik differenziert, wie es aufschlussreich und anschlussfähig für die mediendidaktische Diskussion sein kann. Knapp gefasst könnten OEP im Sinne kollektiver Praktiken unter einem «Primat des Ganzen» gefasst werden, wonach sich jeder «individuelle[r] Akt [...] auf der Basis von Routinen wie von selbst ins Gesamtbild zu fügen» scheint (ebd., 145) ${ }^{9}$. Beschreibt man in Anlehnung an Alkemeyer OEP aber - ähnlich mikrosoziologischer Zugänge - über die (Re-)Konstruktion der verschiedenen Perspektiven der Teilnehmenden, «wird das interaktive Machen einer Praktik in seiner Prozesshaftigkeit» (ebd., 146) in den Fokus gerückt ${ }^{10}$, die konstituierend für eine kollektive Praxis sind.

In einem nächsten Schritt soll, im Vorfeld weiterer Erörterungen bezüglich OEP, der Begriff der Digitalisierung herangezogen werden, um ihn im Kontext von OEP und Hochschule einzuordnen. Es wird im vorliegenden Beitrag von Digitalisierung als einem gesellschaftlichen Prozess ausgegangen, der - neben anderem - auch Prozesse des Lehrens und Lernens, einschliesslich spezifischer Praktiken, inkludiert. Digitalisierung fordert derzeit die Hochschule als Organisation und die darüber eingebundenen Akteurinnen und Akteure heraus und kann zur Transformation der derzeit als eher starr zu identifizierenden Strukturen führen. In diesem Zusammenhang ist Stalder (2018) zuzustimmen, wenn er schreibt:

«Im Zuge der Digitalisierung - der Ausweitung des Einsatzes digitaler Technologien - ist eine neue Infrastruktur der Wahrnehmung, der Kommunikation und der Koordination entstanden. Weil dies grundlegende Dimensionen fast aller individuellen und kollektiven Tätigkeiten sind, lösen die neuen Strukturbedingungen des Handelns - die Digitalität - weitreichende Veränderungen aus. Alte Muster des Denkens und Handelns kommen in die Krise, neue Muster entstehen» (ebd., 8)

und weiter herausstellt, dass gerade die Universitäten und Hochschulen vor der Herausforderung stehen, ihre eigenen Praktiken unter den Bedingungen der Digitalität zu hinterfragen, anzupassen und neu zu entwickeln (vgl. ebd., 14). Folglich wird mit

9 Im vorliegenden Kontext tendenziell in Relation zu einer Open Education und Open Pedagogy, z.B. bei der Awareness für und Schaffung einer Kultur der Offenheit und des Teilens durch Handeln der Akteurinnen und Akteure.

10 Für den vorliegenden Zusammenhang im Sinne einer offenen Praxis bzw. open practices oder gar doing openness z.B. bei der mehr oder weniger freien Lizensierung von Bildungsmedien im Kontext von OER. 
Blick auf den Prozess der sich potenziell verändernden Praktiken statt von E-Learning umfassender von einer Digitalisierung von Lehren und Lernen unter den Bedingungen der Digitalität gesprochen. Dabei wird über Technologien und Infrastruktur hinaus auch den sich entwickelnden Verhältnissen und zu erwartenden Spannungen zwischen bisherigen und neuen Praktiken als soziale und kulturelle Rahmungen Beachtung geschenkt ${ }^{11}$. Praxis meint dann eine transferierende Tätigkeit, d.h. das Anwenden und Erproben von Ideen, Konzepten und Theorien (u.a. Richter und Allert 2017). Unter Praktik bzw. Praktiken ist hier die grundsätzliche Art und Weise der Ausübung einer Tätigkeit zu verstehen, eine Handlungsweise, ein in Aktion sein, ein «doing» oder ein «saying» ${ }^{12}$ vor dem Hintergrund des habitualisierten Handelns der Beteiligten bzw. der Akteurinnen und Akteure (siehe u.a. ausführlicher bei Reckwitz 2003; Hörning und Reuter 2004 ${ }^{13}$ ). Aus praxistheoretischer Perspektive und mit Blick auf Openness im Bildungsbereich lässt sich mit OEP hier anknüpfen: Einerseits über medienpädagogische und mediendidaktische Ansätze und Konzepte als rahmender Kontext (vgl. u.a. für eine partizpative Mediendidaktik Mayrberger in Vorbereitung) und andererseits auf Ebene der Praktiken und Praxis.

Derzeit finden sich in der vorwiegend internationalen Fachdiskussion tendenziell zwei Perspektiven auf OEP: Eine enger an OER orientierte, die OEP nicht ohne OER für realisierbar hält und eine am pädagogischen Konzept einer Open Pedagogy (Hegarty 2015) orientierte Perspektive, die nicht zwingend OER für OEP voraussetzt, sondern eine Open Pedagogy in den Fokus nimmt und u.a. auch Fragen von Machtverhältnissen im Zuge von Openness kritisch würdigt (vgl. für einen Überblick zu der weiten und engen Perspektive auf OEP auf Basis von aktuellen empirischen Analysen Cronin 2017, 2018; Cronin und MacLaren 2018; Koseoglu und Bozkurt 2018).

Für eine Illustration des Diskussionsstands und um für das hier vorliegende Literature Review ein Bild von OEP als Ausgangslage bereit zu stellen, erscheinen nachfolgende exemplarische Perspektiven auf OEP bezeichnend. So wurde bereits im Projektkontext der europäischen Open Educational Quality Initiative (OPAL ${ }^{14}$ ) festge-

11 Solche Spannungsverhältnisse in der (Weiter-)Entwicklung von Praktiken lassen sich auch aus Perspektive des Veränderungsmanagements oder Chance-Managements betrachten, das besonders im Bereich der Digitalisierung in der Bildungsorganisation Hochschule eine ebenso zentrale Rolle spielt. Hier könnte man die (Re-)Evolution von Praktiken durchaus auch aus Perspektive einer organisationalen Ambidextrie reflektieren, bei der es darum geht, Bisheriges und Neues einer Organisation passend zusammen zu bringen (u.a. Rost, Renzl und Kaschube 2014).

12 Siehe dazu Reckwitz (2003): «Wenn man als ,kleinste Einheit‘ des Sozialen nicht ein Normensystem oder ein Symbolsystem, nicht ,Diskurs' oder ,Kommunikation' und auch nicht die ,Interaktion', sondern die ,Praktik' annimmt, dann ist diese kleinste Einheit des Sozialen in einem routinisierten „nexus of doings and sayings" (Schatzki) zu suchen, welches durch ein implizites Verstehen zusammengehalten wird. Genau dies ist eine, soziale Praktik': eine Praktik der Verhandlung, eine Praktik des Umgangs mit einem Werkzeug, eine Praktik im Umgang mit dem eigenen Körper etc.» (ebd., 290).

13 Entsprechend wird die Annahme vertreten, dass ein Anschluss an praxistheoretische Positionen hier hilfreich und sinnvoll sein könnte vgl. Bellinger, Bettinger und Dander (2018); Reckwitz (2003) sowie Bourdieu (u.a. 1998).

14 https://www.icde.org/open-educational-quality-initiative. 
stellt: "Open Educational Practices (OEPs) constitute the range of practices around the creation, use, and management of open educational resources with the intent to improve quality and innovate education» (OPAL 2011a, 4). Das Verständnis von Praktiken wurde weiter wie folgt umschrieben: «practices which support the (re)use and production of OER through institutional policies, promote innovative pedagogical models, and respect and empower learners as co-producers on their lifelong learning path» (OPAL 2011b, 12). Im Zuge ihrer qualitativen Fallstudie kommt Cathrine Cronin (2017) zu folgender Umschreibung von OEP:

"collaborative practices that include the creation, use, and reuse of OER, as well as pedagogical practices employing participatory technologies and social networks for interaction, peer-learning, knowledge creation, and empowerment of learners» (ebd., 18).

Abschliessend soll als dritter Blickwinkel das jüngst veröffentlichte Verständnis von OEP der Educause-Initiative ${ }^{15}$ angeführt werden, die in prägnanter Kürze in ihrer breit rezipierten Short-Paper-Reihe 47 Things You Should Know About» ebenfalls die bisher identifizierte enge oder weite Perspektive auf OEP aufgreift ${ }^{16}$ und zusätzlich die Anbindung von OEP an Open Scholarship herausstellt:

"While educators often initially embrace open educational content as a way to maximize access to curricular materials and significantly reduce their costs, many instructors leverage OER to reconceptualize and improve pedagogy and advance authentic, participatory, engaged learning. One definition describes such open educational practices (OEP) as the 'use/reuse/creation of OER and collaborative, pedagogical practices employing social and participatory technologies for interaction, peer-learning, knowledge creation and sharing, and empowerment of learners.' Open educational practices are seen as a means for students and faculty to develop new approaches to co-creating knowledge, assessing student outcomes, and designing programs. In these and other ways, OEP align with the principles of open scholarship» (Green et al. 2018).

Zusammengenommen bleibt allerdings festzuhalten, dass in allen Beiträgen der Fokus auf Praktiken bzw. Practices nicht hinreichend theoretisch fundiert wird, sondern stellenweise als wesentlicher Aspekt vorausgesetzt, oder mit einem rein pragmatischen Ansatz auf die Realisierung von OEP als zu fördernder Faktor für OER fokussiert wird. Daher bleibt insbesondere mit Blick auf notwendige Forschung in diesem Feld die Frage, aus Perspektive des vorliegenden Beitrags, im Moment unbeantwortet, welche Praktiken genau OEP konstituieren? Ist es hinreichend von der

15 Educause ist eine US-amerikanische non-profit Vereinigung, die die Entwicklung der Hochschulbildung und Hochschulen durch Informationstechnologie bzw. Digitalisierung fördert, https://www.educause. edu/.

16 Siehe ausführlicher unter: https://library.educause.edu/resources/2018/8/7-things-you-should-knowabout-open-education-policies. 
vielzitierten Kollaboration als deren wesentlichen Indikator zu sprechen? Ist es der Prozess der Produktion und des Remix von OER, oder sind es die Handlungsweisen entlang von Prozessen und Infrastrukturen, die das (kulturelle wie technische) Teilen erst ermöglichen? Man könnte sich in dieser fragenden Weise in Anlehnung an Hegarty (2015) beispielsweise entlang der acht Attribute einer Open Pedagogy und ihren Implikationen orientieren, die u.a. vom Teilen von Ideen, Partizipation an einer Gemeinschaft, Vertrauen und Zuversicht sowie der (kritischen) Reflexion der Praktiken selbst spricht. Doch verbliebe man mit derartigen Vorgehensweisen weiterhin auf der konzeptionellen Ebene von OEP, wozu zahlreiche Beiträge bereits vorliegen. Ebenso könnte man auch weiter gehen und fragen, inwiefern es sich bei OEP nicht eher um «learning practices in higher education in relation to a digital participatory culture» handelt, wie es Costa et al. (2018) aus anderer Perspektive untersuchen.

Mit Blick auf mögliche Anknüpfungspunkte in der mediendidaktischen Forschung, erscheint es daher wichtig im Überblick herauszufinden, wie OEP auf Basis bisheriger empirischer Studien beschrieben und erforscht werden und inwiefern praxistheoretische Sichtweisen dabei Berücksichtigung finden, was besonders für die spezifisch mediendidaktische Perspektive der Autorinnen relevant ist. Gerade für medienpädagogische und -didaktische Fragestellungen scheint eine genaue Bestimmung dessen, was unter offenen Bildungspraktiken und einer offenen Bildungspraxis zu verstehen ist, unumgänglich, um dem Phänomen empirisch differenziert begegnen sowie mit konkreten Forschungsprojekten hier anschliessen zu können. Ferner stellt sich aus fachlicher Perspektive die Frage, welche Implikationen bisherige Forschungsergebnisse für die weitere mediendidaktische Forschung - in diesem Kontext mit Fokus auf das akademische Lehren und Lernen bzw. OEP in der Hochschule im Zusammenhang mit Open Scholarship und Open Science (vgl. Mayrberger und Thiemann 2018) - haben und wie hier mit Blick auf den digitalen Transformationsprozess an deutschen Hochschulen angeschlossen werden kann.

\section{Stand der Forschung: Ein systematisches Review}

Vor dem beschriebenen Hintergrund haben die Autorinnen des vorliegenden Beitrags ein systematisches Review in Anlehnung an der von Okoli und Schabram (2010) dokumentierten Methodik erarbeitet, um in einem ersten Schritt den europäischen Forschungsstand zu OEP für den Hochschulkontext zu erfassen und zu analysieren. In diesem Literature Review werden Forschungsarbeiten der letzten zehn Jahre im europäischen Hochschulkontext fokussiert. Nachfolgend werden das methodische Vorgehen und die Ergebnisse vorgestellt sowie anschliessend erörtert.

Bereits im letzten Jahr sind Literature Reviews im Kontext von OEP erschienen, die hier als Forschungsstand gewertet werden können. Der vorliegende Beitrag knüpft an diese Studien an und - das sei bereits in aller Kürze vorweg geschickt 
- erweitert sie durch seine spezifische Perspektive auf Forschungsarbeiten. Dabei geht die Analyse der Beiträge über die Abstracts hinaus und es wurden mehrere einschlägige Datenbanken verwendet, um ein möglichst breites Bild im Kontext der europäischen Hochschulbildung zu erhalten. Die jüngste Publikation von Koseoglu und Bozkurt (2018) gibt einen sehr breiten Überblick über englischsprachige Titel, Abstracts und Stichworte zu Publikationen der letzten zehn Jahre zu OEP, die in der Datenbank Scopus gelistet sind. OEP werden von den Autorinnen und Autoren dabei als digital open educational practice gefasst. Auf Basis ihres Mixed-Method-Ansatzes, der Soziale-Netzwerkanalyen und lexikalische Analysen einschliesst, identifizieren sie drei Diskussionstränge, wovon einer das Verhältnis von OEP und OER betrifft. Sie entwerfen auf Basis ihrer Analyse ein umfassendes Rahmenwerk für OEP im Sinne einer Lernökologie (ebd., 455f.). In einem weiteren Überblicksbeitrag, den die Arbeitsgruppe um Katy Jordan und Martin Weller (2017) zum Thema «Openness» vorlegt, werden vor allem Publikationen rund um ausgewählte Projektförderlinien systematisiert. Die Fragestellung der nachfolgend vorgestellten Ergebnisse grenzen sich auch von Catherine Cronin (2018) ab, die sich im dritten Kapitel ihrer Dissertationsschrift mit Forschungsarbeiten zu OEP in Higher Education unter besonderer Berücksichtigung der theoretischen Ansätze der «Network-Society» von Castells (vgl. 2001) sowie der «Participatory Culture» von Jenkins et al. (vgl 2009; 2015) analytisch auseinandersetzt (vgl. ebd., 26). Eine im Anschluss daran entstandene Studie von Cronin und MacLaren (2018) fokussiert primär die theoretischen Arbeiten zu OEP im Kontext von vier identifizierten zeitlichen Entwicklungsphasen von OEP seit 2006, wenngleich auch hier empirische Arbeiten mit aufgegriffen werden.

Im vorliegenden Beitrag werden nachfolgend solche Arbeiten zu OEP berücksichtigt, die sich auf Basis von nicht-standardisierten wie standardisierten Methoden im weitesten Sinne mit ihren Frage- und Zielstellungen auf Forschungsgegenstände im europäischen Forschungskontext beziehen oder diesen vergleichend berücksichtigen. Hierbei wurden Arbeiten im Kontext von OEP, die sich rein theoretisch oder programmatisch auf OEP beziehen und/oder allein von Praxisprojekten und -erfahrungen berichten, nicht berücksichtigt.

\section{Vorgehen des systematischen Reviews}

Um die Nachvollziehbarkeit zu gewährleisten, wird nun das, für das Systematic Literature Review gewählte, forschungsmethodische Vorgehen nach Okoli und Schabram (2010) nachgezeichnet. Es handelt sich hierbei um ein Vorgehen, welches sowohl als Erhebungs- und Analysemethode gleichermassen gefasst werden kann. Die Autorinnen erachten die vorgeschlagene Abfolge als passend, da hierbei über rein standardisierte Verfahren hinaus gegangen und eine umfassende Textanalyse eingeschlossen wird. 
Eine Systematisierung bzw. methodisch fundierte systematische Übersichtsarbeit erscheint notwendig, da es sich bei der wissenschaftlichen Auseinandersetzung zu OEP an Hochschulen um ein vergleichsweise junges aber - besonders in Anbetracht der zahlreichen bildungs- und hochschulpolitischen Förderungslinien und Initiativen - durchaus expandierendes Forschungsfeld handelt, um die Fülle an Arbeiten mitsamt deren Erkenntnisinteresse überblicken und Forschungslücken identifizieren zu können. Petticrew und Roberts (2006) formulieren diesen Anspruch für die Sozialwissenschaften wie folgt:

«Systematic literature reviews are a method of making sense of large bodies of information, and a means of contributing to the answers of questions about what works and what does not - and many types of other questions too. They are a method of mapping out areas of uncertainty, and identifying where little or no relevant research has been done, but where new studies are needed. Systematic reviews also flag up areas where spurious certainty abounds. These are areas where we think we know more than we do, but where in reality there is little convincing evidence to support our believes» (ebd., 2; Hervorh. im Original).

Methodisch orientiert sich das Vorgehen für das vorliegende Review an vier (Entwicklungs-)Phasen mit jeweils zwei Arbeitsschritten, die von den kanadischen Forschern Chitu Okoli und Kira Shabram (2010) in einem Leitfaden ausführlich beschrieben wurden. In der ersten Phase, dem so genannten Planning, steht die Planung und Arbeitsorganisation im Vordergrund. Zunächst geht es darum, Ziele und Zwecke des angestrebten Reviews zu klären und eine transparente Vorgehensweise bei allen beteiligten Akteurinnen und Akteuren sicherzustellen (vgl. ebd., 14). Übergreifendes Ziel des vorliegenden Reviews ist es, einen systematischen Überblick zu Forschungsarbeiten zu OEP für den europäischen Hochschulkontext zu erarbeiten. Dabei sind folgende spezifische Unterfragen für die Analyse leitend:

- Welche Akteurinnen und Akteure (Lehrende, Lernende, Administration etc.) stehen bei den Forschungsarbeiten im Zentrum des Interesses?

- Welche (Lehr- und Lern-)Szenarien werden als OEP ausgewiesen und empirisch untersucht?

- Wie werden OEP begrifflich definiert und welche theoretischen Ansätze finden dabei Berücksichtigung?

- Inwieweit werden offene Bildungsmaterialien (OER) als Treiber für OEP fokussiert?

- Welcher Begriff von Offenheit steht hinter dem Forschungsvorhaben?

- Welche Forschungsparadigmen, -designs und -methoden finden sich und wie werden die Vorgehensweisen in den einzelnen Arbeiten methodologisch und methodisch begründet?

- Was sind die zentralen Ergebnisse und Schlussfolgerungen der Forschungsarbeiten für den Bildungskontext Hochschule? 
Da mehrere Personen an der Erarbeitung des Reviews ${ }^{17}$ beteiligt waren, wurde die Art und Weise des Vorgehens im Vorfeld partizipativ abgestimmt und mit allen beteiligten Akteurinnen besprochen, wie die Prozessschritte im Einzelnen gemeinsam dokumentiert werden. Es wurde sich im Sinne eines Samples darauf verständigt das Review zeitlich auf veröffentlichte Forschungsarbeiten der letzten zehn Jahre zu konzentrieren und die Reichweite der Recherche grundsätzlich weltweit anzulegen, um in einem ersten Schritt die (ggf. vergleichenden) Arbeiten mit Bezug zum europäischen Hochschulkontext zu identifizieren ${ }^{18}$. In der zweiten Phase, der Selection, stehen die eigentliche Literaturrecherche sowie die Durchsicht der ersten Ergebnisse im Fokus (vgl. ebd., 19). Für den vorliegenden Fall wurde die Literatursuche anfangs auf drei Datenbanken konzentriert: ERIC ${ }^{19}$ und LearnTechLib ${ }^{20}$ als einschlägige Datenbanken für die internationale Forschungscommunity sowie FIS-Bildung ${ }^{21}$ für den vordergründig deutschsprachigen Raum. Zunächst wurde in allen Datenbanken - FIS-Bildung ausgenommen - mit den Schlagworten «Open Education» und «Higher Education» sowie "Open Educational Practice/Open Educational Practices» und «Higher Education» im Zeitraum von 2008 bis 2018 gesucht. In FIS-Bildung wurde mit den Schlagwortkombinationen «Open Educational Practice/Open Educational Practices» und/oder «Hochschule/Universität» sowie "Offene Bildungspraktiken» und/ oder «Hochschule/Universität» unter der zeitlichen Eingrenzung von zehn Jahren, d.h. von 2008 bis Juni 2018, gesucht. Die enorme Fülle an Treffern - exemplarisch: Suche in LearnTechLib für die Schlagworte «Open Education» + «Higher Education» ergab 10.739 Treffer; die Schlagwortsuche «Open Educational Practice/Open Educational Practices»+ «Higher Education» ergab 5.594 Treffer - wurde dann nach Kriterien eingegrenzt, die dem Erkenntnisinteresse des Reviews kongruent sind: So wurden nach dem ersten Suchdurchlauf über die drei Datenbanken hinweg 78 Treffer gefunden, die sich - im weitesten Sinne - forschend mit OEP im Kontext Hochschule befassen. Anschliessend erfolgte das Practical Screening, welches einen zentralen Stellenwert im Vorgehen einnimmt, da hierbei im Sinne einer Auslese bestimmt wird, welche Forschungsarbeiten tatsächlich in das Review aufgenommen werden und welche nicht (vgl. Okoli und Schabram 2010, 21).

Leitende Kriterien für das Screening zur Zusammenstellung des Samples waren vor allem, dass es sich bei den Fundstücken um empirische Studien mit entsprechendem Forschungsdesign handelt, was Textsorten wie rein konzeptionelle, wie programmatische Positionsbestimmungen, Erfahrungs- und Praxisberichte sowie

17 Im diesem Forschungsprojekt wurden die Autorinnen von den studentischen Hilfskräften Amelie Nickel und Cansu Kartoglu unterstützt, denen an dieser Stelle herzlich gedankt sei.

18 Aus forschungsökonomischen Gründen wird die Analyse der umfassenden weltweiten Recherche in aktualisierter Form zu einem späteren Zeitpunkt an anderer Stelle erfolgen.

19 https://eric.ed.gov/.

20 https://www.learntechlib.org/.

21 https://www.fachportal-paedagogik.de/literatur/produkte/fis_bildung/fis_bildung.html. 
Diskussions- und Konzeptpapiere ausschliesst. So wurden insgesamt 46 Studien für den Korpus des weltweiten Reviews identifiziert, wovon 27 Studientreffer aus dem europäischen Forschungskontext stammen. In einem zweiten Suchdurchlauf wurde Scopus $^{22}$ als vierte renommierte Datenbank hinzugezogen. Auch in Scopus wurde mit den Schlagworten "Open Educational Practice/Open Educational Practices» und "Higher Education» sowie unter der zeitlichen Eingrenzung von zehn Jahren gesucht und es ergaben sich 24 Treffer. Anschliessend wurde abgeglichen, welche der 24 Suchtreffer auch in den weiteren Datenbanken (ERIC, LearnTechLib und FIS-Bildung) gefunden werden konnten bzw. herausgefiltert, welche der Dokumente noch nicht berücksichtigt wurden. Somit lieferte die Recherche in Scopus 15 weitere Arbeiten, die dann erneut einem Screening unterzogen wurden. Dadurch konnten zwölf weitere Forschungsarbeiten für das Review identifiziert werden, von denen vier aus dem europäischen Forschungskontext stammen. Die darauf folgende dritte Phase, die Extraction, die von den Autorinnen schon im vorangegangen Screening berücksichtigt wurde, sieht vor, die Studien für das Review entlang der dort umgesetzten forschungsmethodischen Vorgehensweise einzuordnen, um sie anschliessend analysieren zu können (vgl. ebd., 25). Dafür wurden alle 58 Fundstücke aus den beiden Suchdurchläufen in den vier Datenbanken gelesen, entlang der leitenden Fragestellungen exzerpiert und schliesslich einer Feinanalyse unterzogen. Neben den Fragestellungen, der methodischen Vorgehensweise (u.a. Stichprobe, Erhebung sowie Auswertung) sowie den zentralen Ergebnissen der jeweiligen Studien, standen dabei vordergründig der Ausgangspunkt der Untersuchung - zentrale (Vor-)Annahmen einschliesslich Begriffsbestimmungen von Openness und definitorische Bestimmungen von OEP im Zentrum des Interesses. Entsprechend wurden für den vorliegenden Beitrag nun die 31 Forschungsarbeiten (vgl. Tab. 1) zu OEP für den Hochschulkontext aus Europa als Analysebasis herangezogen, die im Folgenden in Form einer Tabelle im Überblick dargelegt werden.

\begin{tabular}{|c|c|}
\hline 2018 & \\
\hline 2017 & $\begin{array}{r}\text { Cronin, Catherine. 2018. Openness and praxis: A situated study of academic staff me- } \\
\text { aning-making and decision-making with respect to openness and use of open educa- } \\
\text { tional practices in higher education. Galway, Irland: NUI Galway. http://hdl.handle. } \\
\text { net/10379/7276. }\end{array}$ \\
\hline & $\begin{array}{l}\text { Challinor, Jane, Victoria I. Marín, und Gemma Tur. 2017. «The development of the re- } \\
\text { flective practitioner through digital storytelling.» International Journal of Technolo- } \\
\text { gy Enhanced Learning 9 (2-3): 186-203. https://doi.org/10.1504/IJTEL.2017.084498. }\end{array}$ \\
\hline $\begin{array}{l}\text { Cronin, Catherine. 2017. «Openness and Praxis: Exploring the Use of Open Educational } \\
\text { Practices in Higher Education». International Review of Research in Open and Distri- } \\
\text { buted Learning 18 (5): 15-34. https://doi.org/10.19173/irrodl.v18i5.3096. }\end{array}$ \\
\hline
\end{tabular}

22 https://www.scopus.com. 


\begin{tabular}{|c|c|}
\hline & $\begin{array}{l}\text { Gallardo, Matilde, Sarah Heiser, und Ximena Arias McLaughlin. 2017. «Developing pe- } \\
\text { dagogical expertise in modern language learning and specific learning difficulties } \\
\text { through collaborative and open educational practices». Language Learning Jour- } \\
\text { nal } 45 \text { (4): } 518-529 \text {. https://doi.org/10.1080/09571736.2015.1010447. }\end{array}$ \\
\hline & $\begin{array}{l}\text { Kaatrakoski, Heli, Allison Littlejohn, und Nina Hood. 2017. «Rethinking professional } \\
\text { learning in higher education: a study on how the use of Open Educational Resour- } \\
\text { ces triggers the adoption of Open Educational Practice». Qwerty - Open and Inter- } \\
\text { disciplinary Journal of Technology, Culture and Education } 12 \text { (2): 46-63. http://www. } \\
\text { ckbg.org/qwerty/index.php/qwerty/article/view/261. }\end{array}$ \\
\hline & $\begin{array}{l}\text { Olsson, Ulf. 2017. «Higher Education Lecturers' Lived Experience of Going Public in } \\
\text { MOOCs». Open Praxis 9(3): 287-297. https://doi.org/10.5944/openpraxis.9.3.589. }\end{array}$ \\
\hline & $\begin{array}{l}\text { Santos-Hermosa, Gema, Núria Ferran-Ferrer, und Ernest Abadal. 2017. «Repositories of } \\
\text { Open Educational Resources: An Assessment of Reuse and Educational Aspects». } \\
\text { International Review of Research in Open and Distributed Learning } 18 \text { (5): 84-120. } \\
\text { https://doi.org/10.19173/irrodl.v18i5.3063. }\end{array}$ \\
\hline \multicolumn{2}{|r|}{ 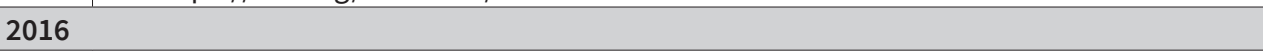 } \\
\hline & $\begin{array}{l}\text { Castaño Muñoz, Jonatan, Yves Punie, Andreia Inamorato dos Santos, Marija Mitic, und } \\
\text { Rita Morais. 2016. «How are Higher Education Institutions Dealing with Openness? } \\
\text { A Survey of Practices, Beliefs, and Strategies in Five European Countries. A Survey } \\
\text { of Practices, Beliefs and Strategies in Five European Countries». JRC Science for } \\
\text { Policy Report, hrsg. v. Institute for Prospective Technological Studies. https://doi. } \\
\text { org/10.2791/709253. }\end{array}$ \\
\hline & $\begin{array}{l}\text { Masterman, Elizabeth. 2016. «Bringing Open Educational Practice to a Research-Inten- } \\
\text { sive University: Prospects and Challenges». The Electronic Journal of e-Learning } 14 \\
\text { (1): } 31-42 \text {. }\end{array}$ \\
\hline & $\begin{array}{l}\text { Nascimbeni, Fabio, und Daniel Burgos. 2016. «In Search for the Open Educator: Propo- } \\
\text { sal of a Definition and a Framework to Increase Openness Adoption Among Univer- } \\
\text { sity Educators». International Review of Research in Open and Distributed Learning } \\
17 \text { (6): 1-17. https://doi.org/10.19173/irrodl.v17i6.2736. }\end{array}$ \\
\hline & $\begin{array}{l}\text { Perryman, Leigh-Anne, und Tim Seal. 2016. «Open Educational Practices and Attitudes } \\
\text { to Openness across India: Reporting the Findings of the Open Education Research } \\
\text { Hub Pan-India Survey». Journal of Interactive Media in Education } 15 \text { (1): 1-17. http:// } \\
\text { doi.org/10.5334/jime.416. }\end{array}$ \\
\hline & $\begin{array}{l}\text { Perryman, Leigh-Anne, und Beatriz de los Arcos. 2016. «Women's empowerment } \\
\text { through openness: OER, OEP and the Sustainable Development Goals». Open Pra- } \\
\text { xis } 8 \text { (2): 163-180. https://doi.org/10.5944/openpraxis.8.2.289. }\end{array}$ \\
\hline \multicolumn{2}{|r|}{ 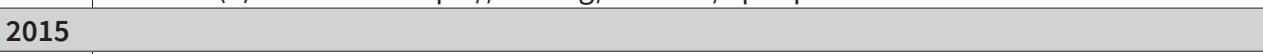 } \\
\hline & $\begin{array}{l}\text { Beaven, Tita. 2015. «OER (re)use and language teachers' tacit professional knowledge: } \\
\text { Three vignettes». In } 10 \text { years of the LLAS elearning symposium: Case studies in good } \\
\text { practice, hrsg. v. Kate Borthwick, Erika Corradini, und Alison Dickens, 77-88. Dub- } \\
\text { lin: Research-publishing.net. https://doi.org/10.14705/rpnet.2015.000269. }\end{array}$ \\
\hline & $\begin{array}{l}\text { Coughlan, Tony, und Leigh-Anne Perryman. 2015. «Learning from the innovative open } \\
\text { practices of three international health projects: IACAPAP, VCPH and Physiopedia». } \\
\text { Open Praxis } 7 \text { (2): 173-189. https://doi.org/10.5944/openpraxis.7.2.188. }\end{array}$ \\
\hline
\end{tabular}




\begin{tabular}{|c|c|}
\hline & $\begin{array}{l}\text { Nerantzi, Chrissi, und Peter Gossman. 2015. «Towards collaboration as learning: evalu- } \\
\text { ation of an open CPD opportunity for HE teachers». Research in Learning Technolo- } \\
\text { gy } 23 \text { (2015): 1-14. https://doi.org/10.3402/rlt.v23.26967. }\end{array}$ \\
\hline \multicolumn{2}{|r|}{ 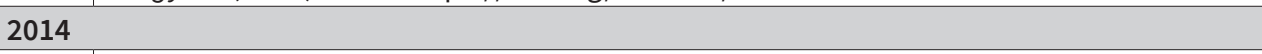 } \\
\hline & $\begin{array}{l}\text { Atenas, Javiera, Leo Havemann, und Ernesto Priego. 2014. «Opening Teaching Land- } \\
\text { scapes: The Importance of Quality Assurance in the Delivery of Open Educational } \\
\text { Resources». Open Praxis } 6 \text { (1): 29-43. http://doi.org/10.5944/openpraxis.6.1.81. }\end{array}$ \\
\hline & $\begin{array}{l}\text { Hassler, Bjoern, Sara Hennessy, Simon Knight, und Teresa Connolly. 2014. «Developing } \\
\text { an Open Resource Bank for Interactive Teaching of STEM: Perspecives of School } \\
\text { Teachers and Teacher Educators». Journal of Interactive Media in Education } 2014 \\
\text { (1): 1-24. https://doi.org/10.5334/2014-09. }\end{array}$ \\
\hline & $\begin{array}{l}\text { Ion, Georgeta, und Romita lucu. 2014. "Professionals' perceptions about the use of } \\
\text { research in educational practice». European Journal of Higher Education 4(4): 334- } \\
\text { 347. https://doi.org/10.1080/21568235.2014.899154. }\end{array}$ \\
\hline & $\begin{array}{l}\text { Kursun, Engin, Kursat Cagiltay, und Gulfidan Can. 2014. «An Investigation of Faculty } \\
\text { Perspectives on Barriers, Incentives, and Benefits of the OER Movement in Turkey». } \\
\text { The International Review of Research in Open and Distance Learning } 15 \text { (6): 14-32. } \\
\text { http://doi.org/10.19173/irrodl.v15i6.1914. }\end{array}$ \\
\hline & $\begin{array}{l}\text { Mtebe, Joel Samson, und Roope Raisamo. 2014. «Challenges and instructors' intention } \\
\text { to adopt and use open educational resources in higher education in Tanzania». The } \\
\text { International Review of Research in Open and Distributed Learning } 15 \text { (1): 250-271. } \\
\text { http://doi.org/10.19173/irrodl.v15i1.1687. }\end{array}$ \\
\hline & $\begin{array}{l}\text { Mtebe, Joel Samson, und Roope Raisamo. 2014. «Investigating perceived barriers to } \\
\text { the use of open educational resources in higher education in Tanzania». The Inter- } \\
\text { national Review of Research in Open and Distributed Learning } 15 \text { (2): 43-66. http:// } \\
\text { doi.org/10.19173/irrodl.v15i2.1803. }\end{array}$ \\
\hline & $\begin{array}{l}\text { Perryman, Leigh-Anne, und Tony Coughlan. 2014. «When Two Worlds Don`t Collide: } \\
\text { Can Social Curation Address the Marginalisation of Open Educational Practices and } \\
\text { Resources from Outside Academia?» Journal of Interactive Media in Education } 2014 \\
\text { (2: 3): } 1 \text {-12. http://doi.org/10.5334/jime.ab. }\end{array}$ \\
\hline & $\begin{array}{l}\text { Schreurs, Bieke, Antoine Van den Beemt, Fleur Prinsen, Gabi Witthaus, Gráinne Cono- } \\
\text { le, und Maarten De Laat. 2014. "An Investigation into Social Learning Activities by } \\
\text { Practitioners in Open Educational Practices». International Review of Research in } \\
\text { Open and Distance Learning 15 (4): 1-20. http://doi.org/10.19173/irrodl.v15i4.1905. }\end{array}$ \\
\hline \multicolumn{2}{|r|}{ r } \\
\hline & $\begin{array}{l}\text { Alvarez, Inma, Tita Beaven, und Anna Comas-Quinn. 2013. «Performing Langua- } \\
\text { ges: an example of integrating open practices in staff development for language } \\
\text { teachers». Journal of e-Learning and Knowledge Society } 9 \text { (1): 85-92. https://doi. } \\
\text { org/10.20368/1971-8829/804. }\end{array}$ \\
\hline & $\begin{array}{l}\text { Beaven, Tita. 2013. «Use and Reuse of OER: professional conversations with language } \\
\text { teachers». Journal of e-Learning and Knowledge Society } 9 \text { (1): 59-71. https://doi. } \\
\text { org/10.20368/1971-8829/802. }\end{array}$ \\
\hline
\end{tabular}




\begin{tabular}{|c|c|}
\hline & $\begin{array}{l}\text { Borthwick, Kate, und Angela Gallagher-Brett. 2013. "Inspiration, ideas, encourage- } \\
\text { ment': teacher development and improved use of technology in language teaching } \\
\text { through open educational practice». Computer Assisted Language Learning } 27 \text { (2): } \\
\text { 163-183. https://doi.org/10.1080/09588221.2013.818560. }\end{array}$ \\
\hline & $\begin{array}{l}\text { Coughlan, Tony, und Leigh-Anne Perryman. 2013. «Beyond the Ivory Tower: A Model for } \\
\text { Nurturing Informal Learning and Development Communities through Open Educa- } \\
\text { tional Practices». International Journal of Educational Technology in Higher Educa- } \\
\text { tion } 10 \text { (1): 312-326. https://doi.org/10.7238/rusc.v10i1.1586. }\end{array}$ \\
\hline & $\begin{array}{l}\text { Perryman, Leigh-Anne, und Tony Coughlan. 2013. «The realities of 〈reaching out〉: enac- } \\
\text { ting the public-facing open scholar role with existing online communities». Journal } \\
\text { of Interactive Media in Education } 2013 \text { (1: 21): 1-14. http://doi.org/10.5334/2013-21. }\end{array}$ \\
\hline \multicolumn{2}{|r|}{ e } \\
\hline & $\begin{array}{l}\text { Banzato, Monica. 2012. «A Case Study of Teachers' Open Educational Practices». Journal } \\
\text { of e-Learning and Knowledge Society } 8 \text { (3): 153-163. https://doi.org/10.20368/1971- } \\
8829 / 650 \text {. }\end{array}$ \\
\hline & $\begin{array}{l}\text { Neuhoff, Antje. 2012. "Shared Practices amongst Teachers in Online Training Cour- } \\
\text { ses». Journal of e-Learning and Knowledge Society } 8 \text { (3): 105-118. https://doi. } \\
\text { org/10.20368/1971-8829/646. }\end{array}$ \\
\hline & $\begin{array}{l}\text { Rolfe, Vivien. 2012. «Open educational resources: staff attitudes and awareness». } \\
\text { Research in Learning Technology } 20 \text { (2012): 1-13. https://doi.org/10.3402/rlt. } \\
\text { v20i0.14395. }\end{array}$ \\
\hline
\end{tabular}

Tab. 1.: Chronologische Auflistung der Forschungsarbeiten aus dem Korpus des systematischen Reviews zu OEP für den Hochschulkontext für Europa bis Juni 2018 - beginnend mit der jüngsten Veröffentlichung.

Vor dem Hintergrund der für das systematische Review leitenden Fragestellungen werden die nunmehr identifizierten Forschungsarbeiten, die zugleich als Forschungsstand zu OEP im Hochschulkontext für Europa gelten können und einen Teil der Ergebnisse ausmachen, nachfolgend analysiert und abschliessend aus einer mediendidaktischen Perspektive diskutiert.

\section{Ergebnisse des Reviews und Diskussion für den europäischen Forschungskontext}

Die Feinanalyse sieht die Synthese der im Korpus des Reviews enthaltenen Forschungsarbeiten vor (vgl. Okoli und Schabram 2010, 30). Für den vorliegenden Fall wurden die 31 Studien $^{23}$ zu OEP aus dem europäischen Forschungskontext entlang der leitenden Fragestellungen des Reviews analysiert und anschliessend dimensioniert. Die Dimensionierung erfolgte auf Basis der unterschiedlichen (Begriffs-)

\footnotetext{
23 Im Rahmen der Feinanalyse wurde eine Forschungsarbeit von Georgeta Ion und Romita Icu (2014) aus dem Korpus entnommen, da sich herausstellte, dass die Studie weder Openness noch OEP explizit oder implizit berücksichtigt, sondern den «State of the Art» der Bildungsforschung in Rumänien nachzeichnet (Quelle: Ion, G. und lucu, R. 2014. "Professionals' perceptions about the use of research in educational practice». European Journal of Higher Education 4(4): 334-347. https://doi.org/10.1080/21568235.2014.899154.)
} 
Verständnisse von Offenheit bzw. Openness und OEP, die den Studien mehr oder minder explizit zugrunde lagen. Die Autorinnen erarbeiteten während der Feinanalyse vier Dimensionen von «eher sehr weit» bis «sehr eng» und verfassten zu jeder dieser Dimensionen Arbeitsthesen, um die Studien entsprechend kategorisieren zu können. Aufgrund der gebotenen Kürze soll die weitere Ergebnisdarstellung entlang dieser Dimensionierung erfolgen, da diese ebenfalls Teil des Ergebnisses sind. Vorweg sei darauf hingewiesen, dass die Kategorisierung an dieser Stelle nicht starr zu verstehen ist, da einige Studien bei anderer Schwerpunktsetzung mitunter auch einem anderen Dimensionsabschnitt zugeordnet werden könnten. Entscheidend für die Dimensionierung im Rahmen des vorliegenden Reviews war der Fokus und die Zielsetzung, welche die Autorinnen und Autoren für die jeweilige Untersuchung forcierten.

Hinter dem ersten Dimensionsabschnitt «eher sehr weit» liegt die Arbeitsthese, dass Offenheit oder Openness einen Rahmen seitens der Institution und (Bildungs-) Politik (Governance) braucht, damit Prinzipien einer Open Pedagogy (vgl. Hegarty 2015) für den formalen Bildungskontext Hochschule adaptiert und OEP folglich realisierbar werden. Zwölf der Fundstücke aus dem Gesamtkorpus konnten diesem ersten Dimensionsabschnitt zugeordnet werden. So u.a. die Untersuchung von Castaño Muñoz et al. (2016), die sich mit Fragen zur Adaption von Open Education in fünf europäischen Ländern (Deutschland, England, Frankreich, Polen und Spanien) befassten. Zur Kontextualisierung sei angemerkt, dass die Untersuchung Teil der «Science for Policy Report»-Reihe des Joint Research Centre (JRC) der Europäischen Kommission ist, weshalb der Fokus im Fall dieser quantitativ-ausgerichteten Umfrage, an der sich 178 Hochschulen aus Europa beteiligten, auf dem Zugang zu offenen Materialien mittels digitaler Technologien liegt, was sich im Verständnis von Open Education wiederspiegelt, wenn die Autoren schreiben «Open Education is understood in this study as a mode of realising education using digital technologies to provide alternative and less restrictive access routes to formal and non-formal education» (ebd., 4). Die Autoren kommen zum Schluss, dass offene Lehr-/Lernmaterialien in den fünf untersuchten Ländern mehr oder weniger verbreitet sind und es folglich notwendig scheint, länderübergreifend für Open Education zu sensibilisieren, damit bestehende Mechanismen und Praktiken verändert werden können (vgl. ebd., 5). Eine weitere Mixed-Methods-Studie von Monica Banzato (2012), die eine Teilstudie aus einem grösseren Projekt darstellt, aus dem ein OER-Repository erwachsen soll, fokussierte Bedarfe der Endnutzer, um OEP für die eigene Lehre zu adaptieren; im vorliegenden Fall Lernende und Lehrende der Bildungswissenschaften in Italien. Das der Untersuchung zugrundeliegend begriffliche Verständnis orientiert sich an den Definitionen, die von der OPAL-Initiative sowie Conole (2010) in den Diskurs eingeführt wurden und einen Shift von der reinen OER-Verwendung hin zu OEP stark machen: 
"In order to understand the new educational practices, it is important to shift attention from research into the resources themselves (OER) towards the practices connected with the creation, utilisation and management of OER, defined as Open Educational Practices (OEP) (Conole, 2010)». (Bazanto 2012, 154)

Auch diese Studie, bei der 176 Personen quantitativ und qualitativ befragt wurden, kann dem Dimensionsabschnitt «eher sehr weit» zugeordnet werden, da die Zielsetzung - der Aufbau eines Repositories - leitend war und in der Untersuchung deutlich wurde, dass eine weitreichende Sensibilisierung seitens der Hochschulen für Openness und OEP notwendig ist, da die Lehrenden und Lernenden der Bildungswissenschaften weitestgehend über ein «traditional view of the practices of teaching and learning» (ebd., 160) verfügen, was sich in der verhaltenen Haltung gegenüber der Nutzung und Weiterentwicklung von OER in akademische Bildungszusammenhängen widerspiegelt.

Dem zweiten Dimensionsabschnitt «eher weit» liegt die These zugrunde, dass OEP für den formalen Bildungskontext Hochschule mit offenen und geschlossen Materialien forciert werden können, da die Offenheit des didaktischen Szenarios entscheidend ist. Neun der Fundstücke des Gesamtkorpus konnten diesem Dimensionsabschnitt zugordnet werden; u.a. eine Studie von Neuhoff (2012), die geteilte Unterrichtspraktiken und soziale Interaktionen im Rahmen einer wissenschaftlichen Weiterbildung für Lehrende von Sprachkursen anhand von Kommentaren und Forenbeiträgen im veranstaltungsbegleitenden LMS untersuchte. Der Mehrwert des sozialkollaborativen Austauschs der Teilnehmenden zu Unterrichtspraktiken stand im Fokus der Analyse und die Autorin definierte OEP in Anlehnung an die OPAL-Initiative (2011a) als kollaborative Praktiken (ebd., 106). Final kommt Neuhoff zu dem Ergebnis, dass soziale Interaktion und ferner der Austausch zu und über Unterrichtspraktiken in online Lernumgebungen nicht natürlich erwächst, sondern durch tutorielle Begleitung angeregt werden muss. Eine weitere Studie, die dem Dimensionsabschnitt "eher weit» zugeordnet wurde, ist die Fallstudie von Challinor et al. (2017) zur Methode Digital-Storytelling in der Berufspädagogik mit Schwerpunkt Gesundheits- und Sozialwesen. Hierbei erstellten die Lernenden jeweils zwei digitale Artefakte, durch die sie Aspekte des beruflichen und persönlichen Lernens reflektierten sollten und wurden dazu ermutigt, OER dafür zu verwenden. Der Ansatz der reflexiven Praxis von Donald A. Schön (1983) sowie die von Ala-Mutka, Punie und Redecker (2008) formulierte digital literacy waren dabei für die Forscherinnen leitend. Untersucht wurden insgesamt fünf Fälle von Studierendengruppen (under- und postgraduate) aus England und Spanien, wobei die Reflexionsebenen von Moon (1999) für die Analyse der digitalen Artefakte leitend waren. Die Autorinnen stellen fest, dass die Methode Digital-Storytelling eine gute Möglichkeit darstellt die Lernenden an OER heranzuführen und definierten final in Anlehnung an Linders (2012) eine Philosophie von Openness, 
für das Gesundheits- und Sozialwesen: «Openness as a philosophy is strongly aligned to ideas of citizen empowerment in health and social care, which manifests itself in the increased use of open platforms for campaigning and information exchange by those with care and support needs» (Challinor et al. 2017, 14f.).

Hinter dem dritten Dimensionsabschnitt «eher eng» liegt die These, dass OEP sich immer auf die Einbindung offener Materialien, also OER, bezieht und offene Praktiken ohne diese nicht erwachsen können. Kaatrakoski et al. (2017) haben eine Untersuchung durchgeführt, die eine Teilstudie des ExplOERer-Projekts ist. Rekurrierend auf Theorien selbstregulierten Lernens (Zimmermann 2000), den Ansatz der kulturhistorischen Aktivitätstheorie (Engeström 1987; Leont'ev 1978; Vygotsky 1978) und unter Berücksichtigung des linguistischen Analyserahmens der «Discursive manifestations of contradictions» (Engeström 1999) analysierten die Autorinnen Herausforderungen, denen Lehrende gegenüberstehen, wenn sie ihre eigene Lehr-Lernpraxis in Richtung OEP entwickeln wollen. Die Datenerhebung erfolgte in zwei Phasen: Zunächst wurde eine Umfrage zu Nutzungsweisen von OER durchgeführt ( $N=521)$ und anschliessend wurden 30 halbstrukturierte Interviews mit Lehrenden aus acht verschiedenen Ländern geführt, bei denen Fragen zu OEP durch OER fokussiert wurden. Die Ergebnisse der Teilstudie zeigen, dass die Lernenden durch die Adaption von OEP aktiver wahrgenommen werden, seitens der Lehrenden aber Spannungen hinsichtlich der Einbindung von OER erkennbar sind. Die Autorinnen resümieren folglich, dass Lehrende Richtlinien und Leitbilder benötigen: «Educators need support in evolving their educational practice towards OEP by allowing them to feedback what this change means for their practice and figuring out how to meaningfully move forward» (Kaatrakoski et al. 2017, 59). Eine weitere Forschungsarbeit, die diesem Dimensionsabschnitt zugeordnet werden kann, ist die Untersuchung von Beaven (2013), die die Verwendung und Wiederverwendung von fachspezifischen OER aus dem LORO-Repository für den Sprachenunterricht (auch Fernstudium) explorierte. Die Autorin beobachtete acht Lehrende bei der Unterrichtsvorbereitung und führte zwölf strukturierte Experteninterviews, die Fragen zur Verwendung und Handhabung des Repositories, Erläuterungen zum Vorgehen bei der Lehrvorbereitung und Fragen zu technischen Fähigkeiten der Lehrenden beinhalteten. Die Daten wurden dann inhaltsanalytisch nach Braun und Clarke (2006) ausgewertet. Die Ergebnisse zeigen, dass es bei den Befragten viele offene Lehr-/Lernpraktiken gibt, die nicht unbedingt sichtbar sind: «...as some of the reuse and sharing is not necessarily visible, because it is not published in the OER repositories the OER originally come from, but happens through other informal or more private ways» (Beaven 2013, 68f.). Die Autorin kommt folglich zum Schluss, dass der Austausch von OER essentiell scheint, um den Shift zu OEP zu ermöglichen. 
Dem vierten Dimensionsabschnitt «sehr eng» liegt die Arbeitsthese zugrunde, dass mit OEP bestimmte (medien-)didaktische Szenarien (bspw. MOOCs) gemeint sind, bei denen OER zum Einsatz kommen. Schreurs et al. (2014) versuchten in ihrer qualitativ-interpretativ ausgerichteten Studie offene Praktiken in ihrer Konfiguration zu verstehen und dahingehend zu ergründen, wie Lehrende zusammenarbeiten, damit OEP erwachsen. OEP wird von den Autorinnen und Autoren definiert als «set of activities and support around the creation, use, and repurposing of open educational resources (OERs) and MOOCs» (ebd., 6). Die Untersuchung wurde als komparative Fallanalyse von insgesamt sechs Initiativen aus dem POERUP-Projekt angelegt, bei der 18 Interviews mit Personen aus den Initiativen geführt wurden, die anschliessend nach Glaser und Strauss (2009) ausgewertet wurden. Die Ergebnisse verdeutlichen, dass es verschiedene Ebenen der Partizipation an OEP über die Fälle hinweg gibt und schlussfolgern, dass das Bestreben einer Teilnahme an offenen Bildungspraktiken traditionelle Formen akademischer Laufbahnentwicklungen - hier am Beispiel der Frage nach geistigem Eigentum - zunehmend in Frage stellt (Schreurs et al. 2014, 16).

Zusammenfassend lassen sich die vier Dimensionen wie folgt auf den Punkt bringen:

- OEP im Sinne «eher sehr weit» umfasst in erster Linie die uneingeschränkte Übernahme der Grundsätze einer Open Pedagogy, einschliesslich der institutionellen Rahmenbedingungen und Governance (institutionelle Meso- und Makroebene). Das schliesst die Übernahme der Prinzipien offener Bildung, wie Open Scholarships, die Schaffung offener Online-Lernumgebungen, die auf OER oder Variationen von Open-Labs (DeRosa und Blickensderfer 2017) oder Edu-Labs und OERLabs ausgerichtet sind (z.B. Mayrberger 2018) ein.

- OEP im Sinne «eher weit» umfasst vor allem offene pädagogisch-partizipative Lernszenarien und die Verwendung von OER sowie die Verwendung von urheberrechtlich lizenziertem Unterrichtsmaterial (institutionelle Mikroebene). Damit sind Szenarien, die offene Unterrichtspraktiken oder partizipatives Lernen forcieren, aber in einem geschlossenen Lernmanagementsystem (LMS) der Institution stattfinden oder mit Social-Media-Tools wie Blogs oder Wiki-Software angereichert werden, die auf institutionellen oder ausländischen Servern liegen, eingeschlossen.

- OEP im Sinne «eher eng» bezieht sich auf die Produktion, Nutzung sowie das Remixen von OER entlang der $5 \mathrm{R}$ als Aktivitäten in geeigneten Lernszenarien (ähnlich der «OER-Enabled Pedagogy» von Wiley und Hilton 2018). Auf Mikroebene der Institution umfasst dies bspw. die Co-Kreation von Wissensgegenständen bei der Verwendung und Erstellung von OER während der Arbeit mit offenen Lehrbüchern oder auch das (Um-)Schreiben von Wikipedia-Artikeln als Teil der Bewertung.

- OEP im Sinne "sehr eng» umfasst die Verwendung von OER in offenen oder geschlossenen Lernszenarien, wie bspw. im Kontext von MOOCs oder anderen Formen von Online-Kursen (institutionelle Mikroebene). 
Folgerungen und Ausblick: Eine definitorische Annährung für die mediendidaktische Forschung aus praxistheoretischer Perspektive

Die Ergebnisdarstellung entlang der vier Dimensionen verdeutlicht, wie OEP als Forschungsgegenstand - über die vergangenen zehn Jahre hinweg - in Europa vielfältig untersucht wurde. Eine Vielzahl der Forschungsarbeiten aus dem Korpus des Reviews entstammt grossen Projekten, in denen vorwiegend der Versuch unternommen wurde, die Adaption von OEP (durch den Einsatz von OER) zu explorieren, um zu verstehen, wie sich diese offenen Praktiken in konkreten Lehr-Lernsettings tatsächlich konstituieren. Begriffsverständnisse und definitorische Annährungen sind über die 30 Studien hinweg sehr facettenreich und ähneln sich vor allem dann, wenn die jeweiligen Studien aus einem grossen Forschungsverbund oder Projekt stammen.

Ähnlich der Ergebnisse anderer Literature Reviews, die sich dem Verhältnis von OER und OEP widmeten, wird auch hier zwischen engen und weiten Dimensionen unterschieden. Eine Konkretisierung der jeweiligen Dimensionen als spezifische Praktik und Praxis wäre dann ein fruchtbarer Gegenstand eines anschliessenden (nicht nur) mediendidaktischen Forschungsvorhabens. Es zeigt sich, an diesem Analyseergebnis und über alle Literature Reviews zur Konstitution einer OEP hinweg, wie zentral der Praxisbegriff ist bzw. sein könnte und wie eine praxistheoretische Perspektive hierbei zum Verständnis und zur Erklärung des Feldes beitragen kann. Die Reflexion des Praxisbegriffs stellt einen wesentlichen Ausgangspunkt für theoretische und empirische Überlegungen im Zuge der medienpädagogischen und mediendidaktischen Auseinandersetzung mit OEP im Hochschulkontext dar und die Theorien sozialer Praktiken (vgl. Reckwitz 2003) können hier wertvolle Anknüpfungspunkte liefern. Praxistheoretische Positionen tragen dem Umstand Rechnung, dass das Handeln von Akteurinnen und Akteuren - hier in Bildungszusammenhängen - auf habitualisierte Dispositionen zurückgeht, Handeln und Habitus also untrennbar verwoben sind. Entsprechend erscheint es gerade aus theoretischer wie empirischer Perspektive relevant, OEP unter dieser theoretischen Brille in den Blick zu nehmen und zu ergründen, welche (Bildungs-)Praktiken im Kontext von Openness bislang vorherrschend sind, wie diese erwachsen und welche mediendidaktischen und partizipationsfördernden Gestaltungselemente der Lernumgebungen im weitesten Sinne dabei unterstützen können, den Kulturwandel an Hochschulen unter den Bedingungen von Digitalität und Digitalisierung zu gestalten (vgl. Mayrberger 2014, 2017 sowie in Vorbereitung). Um eine entsprechende mediendidaktische Forschungsperspektive zu verfolgen, wird auf Grund der hier identifizierten Leerstellen von den Autorinnen die folgende praxistheoretisch informierte Begriffsbestimmung von OEP vorgeschlagen:

OEP sind - unter einer praxistheoretischen Perspektive - als partizipative mediendidaktische Gestaltungs-Praxis an Hochschulen zu fassen, welche Lehrende und Lernende gleichermassen als Akteure offener Lehr-Lernpraktiken und -architekturen versteht. Weiter sind OEP im Anschluss an die Theorien sozialer Praxis ein «typisiertes, 
routinisiertes und sozial «verstehbares` Bündel von Aktivitäten» (Reckwitz 2003, 289), das auf der Grundlage inkorporierten Wissens einer impliziten Logik folgt (Bourdieu 1979 und 1993) und sich in spezifischen Formen des Zusammenwirkens von Handlungen, Körpern und (medialen) Artefakten, respektive OER, manifestiert. OEP werden damit sowohl vonseiten inkorporierter als auch in Artefakten materialisierter Wissensbestände her begriffen (Reckwitz 2006, 707), die das Handeln anleiten. Insofern stellen sich OEP als Handlungsvollzüge dar, die ihre Sinnhaftigkeit erst im Vollzug innerhalb eines bestimmten sozialen Kontextes erkennen lassen und einer Logik der Praxis folgen (Bourdieu 1998), die im Zuge der (Hochschul-)Sozialisation erworben wurde.

Diese begriffliche Ausgangslage eröffnet theoretisch und methodologisch vielfältige Anknüpfungspunkte und ist ferner international anschlussfähig bzw. beitragsfähig, weil einerseits das Thema OEP derzeit mit grossem Interesse bearbeitet wird und zum anderen die hier vorgestellte theoretische Hintergrundfolie von OEP noch eine Leerstelle darstellt. Die vorgeschlagene Begriffsbestimmung umfasst gängige Merkmale von OEP, die sich im Kern entlang der vier identifizierten Dimensionen wiederholen und aus spezifischer Perspektive einen konzeptionellen Anker in der partizipativen Mediendidaktik wie theoretischen Anker in den Theorien sozialer Praktiken hat. Ebenso scheint es vor dem Hintergrund der Kenntnis einschlägiger OER- bzw. OEP-Projektaktivitäten augenscheinlich, dass die Begriffsbestimmung mit dem Gegenstand nicht im Widerspruch steht und eine fruchtbare Basis für weitere empirische Untersuchungen in dieser Richtung bietet.

Anders als Koseoglu und Bozkurt $(2018,456)$, die abschliessend feststellen, dass OEP «as a common descriptor for diverse open practices» genutzt werde und deshalb vorschlagen «we can at least established a shared understanding of where research is at within the field of education and across disciplines and academic communities», wird hier eine proaktivere und entwicklungsorientiertere Perspektive für erstrebenswerter gehalten. Aufgrund des zunehmenden Interesses und der Breite des Felds um OEP, soll die vorgeschlagene Forschungsperspektive verhelfen OEP systematisch zu bearbeiten, um zu einer immer trennschärferen theoretisch wie empirisch abgesicherten Dimensionierung von OEP für den Bildungskontext zu gelangen.

Dabei geht es nicht allein darum eine allumfassende Begriffsbestimmung zu formulieren, sondern diese entlang der Dimensionierungsidee weiter zu erarbeiten, sodass sie die gesamte Breite der offenen Bildungspraxis adäquat abbildet. Eine solche Schärfung trägt weiter dazu bei, die derzeitigen, eher als diffus erlebten, Transformationsprozesse und Manifestationen eines Kulturwandels in den (nicht nur) akademischen offenen Praktiken konkreter begreifen und fassen zu können, damit sich zeitgemässe wie in vielerlei Hinsicht passende offene Aus- und Bildungskonzepte für eine zukünftige Handlungsfähigkeit der Akteurinnen und Akteure fundiert entwickeln können sowie theoretische und empirische Arbeiten hier anschliessen können. 


\section{Literatur}

Ala-Mutka, Kirsti, Yves Punie, und Christine Redecker. 2008. «Digital Competence for Lifelong Learning». Luxembourg: Office for Official Publications of the European Communities. http://ftp.jrc.es/EURdoc/JRC48708.TN.pdf.

Alkemeyer, Thomas. 2017. «Praktiken und Praxis. Zur Relationalität von Ordnungs- und SelbstBildung in Vollzügen» In Performance und Praxis. Praxeologische Erkundungen in Tanz, Theater, Sport und Alltag, hrsg. v. Gabriele Klein und Hanna Göbel, 141-165. Bielfeld: transcript. https://doi.org/ 10.14361/9783839432877-006.

Banzato, Monica. 2012. «A Case Study of Teachers' Open Educational Practices.» Journal of e-Learning and Knowledge Society 8 (3): 153-163. https://doi.org/10.20368/1971-8829/650.

Beaven, Tita. 2013. «Use and Reuse of OER: professional conversations with language teachers.» Journal of e-Learning and Knowledge Society 9 (1): 59-71. https://doi.org/10.20368/1971$8829 / 802$.

Bellinger, Franziska, Patrick Bettinger, und Valentin Dander. 2018. «Researching Open Educational Practices (OEP) ». MedienPädagogik: Zeitschrift für Theorie Und Praxis Der Medienbildung 32 (Oktober): 108-121. https://doi.org/10.21240/mpaed/32/2018.10.27.X.

Bourdieu, Pierre. 1993. Sozialer Sinn. Kritik der theoretischen Vernunft (7. Aufl.). Frankfurt am Main: Suhrkamp.

Bourdieu, Pierre. 1979. Entwurf einer Theorie der Praxis auf der ethnologischen Grundlage der kabylischen Gesellschaft. Frankfurt am Main: Suhrkamp.

Bourdieu, Pierre. 1998. Praktische Vernunft - Zur Theorie des Handelns. Frankfurt am Main: Suhrkamp.

Braun, Virginia, und Victoria Clarke. 2006. «Using Thematic Analysis in Psychology». Qualitative Research in Psychology 3 (2): 77-101. https://doi.org/10.1191/1478088706qp063oa.

Cape Town Open Education Declaration. 2007, 2017. Verfügbar unter: http://www.capetowndeclaration.org/.

Castaño Muñoz, Jonatan, Yves Punie, Andreia Inamorato dos Santos, Marija Mitic und Rita Morais. 2016. «How are Higher Education Institutions Dealing with Openness? A Survey of Practices, Beliefs, and Strategies in Five European Countries. A Survey of Practices, Beliefs and Strategies in Five European Countries.» JRC Science for Policy Report, hrsg. v. Institute for Prospective Technological Studies. https://doi.org/10.2791/709253.

Castells, Manuel. 2001. Das Informationszeitalter I. Der Aufstieg der Netzwerkgesellschaft. Opladen: Leske + Budrich Verlag.

Challinor, Jane, Victoria I. Marín, und Gemma Tur. 2017. «The development of the reflective practitioner through digital storytelling.» International Journal of Technology Enhanced Learning 9 (2-3): 186-203. https://doi.org/10.1504/IJTEL.2017.084498.

Conole, Gráinne. 2010. «What would learning in an open world look like? A vision for the future». Keynote gehalten auf der Edmedia conference, Toronto, Juli 1. http://oro.open. ac.uk/22889/. 
Costa, Cristina, Mark Murphy, Ana Lucia Pereira, und Yvette Taylor. 2018. «Higher education students experiences of digital learning and (dis)empowerment.» Australasian Journal of Educational Technology 34 (3): 140-152. https://doi.org/10.14742/ajet.3979.

Cronin, Catherine. 2017. «Openness and Praxis: Exploring the Use of Open Educational Practices in Higher Education». International Review of Research in Open and Distributed Learning 18(5): 15-34. https://doi.org/10.19173/irrodl.v18i5.3096.

Cronin, Catherine. 2018. Openness and praxis: A situated study of academic staff meaning-making and decision-making with respect to openness and use of open educational practices in higher education. Galway, Irland: NUI Galway. http://hdl.handle.net/10379/7276.

Cronin, Catherine, und Iain MacLaren. 2018. «Conceptualising OEP: A review of theoretical and empirical literature in Open Educational Practices». Open Praxis 10 (2): 127-143. http:// dx.doi.org/10.5944/openpraxis.10.2.825.

Deimann, Markus. 2018. "OER-Forschung - Warum es sie bisher nicht gab und wie sich das ändern kann». Synergie - Fachmagazin für Digitalisierung in der Lehre 5: 70-76. https://www. synergie.uni-hamburg.de/de/media/ausgabe05/synergie05-beitrag13-deimann.pdf.

DeRosa, Robin, und Dan Blickensderfer. 2017. «What Makes an Open Lab «Open`?» Essay. Inside Higher Ed (blog). 2. Mai 2017. https://www.insidehighered.com/views/2017/05/02/keyprinciples-open-labs-essay.

Engeström, Yrjö. 1987. Learning by expanding. An activity-theoretic approach to developmental research. 1. Aufl. Helsinki: Orienta-Konsultit Oy.

Engeström, Yrjö. 1999. «Communication, Discourse and Activity». The Communication Review 3 (1-2): 165-85. https://doi.org/10.1080/10714429909368577.

Glaser, Barney G., und Anselm L. Strauss. 2009. The Discovery of Grounded Theory: Strategies for Qualitative Research. New Brunswick; London: Aldine Transaction.

Green, Cable, Barbara Illowsky, David Wiley, David Ernst, Lisa Young, Amanda Coolidge, Robin DeRosa, und Rajiv Jhangiani. 2018. "7 Things You Should Know About Open Education: Policies». https://library.educause.edu/ /media/files/library/2018/8/eli7159.pdf.

Hegarty, Bronwyn. 2015. «Attributes of Open Pedagogy: A Model for Using Open Educational Resources». Educational Technology 55(4: July- August): 3-13. https://www.jstor.org/stable/44430383.

Hörning, Karl H., und Julia Reuter. 2004. «Doing Culture. Kultur als Praxis.» Forum Qualitative Sozialforschung/ Forum: Qualitative Social Research 7(2), Art. 10: 1-15. https://www. transcript-verlag.de/media/pdf/19/49/26/ts243_1.pdf.

Jenkins, Henry, Mizuko Ito, und danah boyd. 2015. Participatory culture in a networked era: a conversation on youth, learning, commerce, and politics. Cambridge, UK; Malden, MA: Polity Press. 
Jenkins, Henry, Ravi Purushotma, Margaret Weigel, Katie Clinton, und Alice J. Robison. 2009. Confronting the challenges of participatory culture: media education for the 21st century. The John D. and Catherine T. MacArthur Foundation Reports on Digital Media and Learning. Cambridge, MA: The MIT Press. https://mitpress.mit.edu/books/confronting-challengesparticipatory-culture.

Jordan, Katy, und Martin Weller. 2017. Openess and education: a beginner's guide. http://oro. open.ac.uk/53028/1/openreclaim-171102172851.pdf.

Kaatrakoski, Heli, Allison Littlejohn, und Nina Hood. 2017. «Rethinking professional learning in higher education: a study on how the use of Open Educational Resources triggers the adoption of Open Educational Practice.» Qwerty - Open and Interdisciplinary Journal of Technology, Culture and Education 12 (2): 46-63. http://www.ckbg.org/qwerty/index.php/ qwerty/article/view/261.

Koseoglu, Suzan, und Aras Bozkurt. 2018. «An exploratory literature review on open educational practices.» Distance Education 39 (4): 441-461. https://doi.org/10.1080/01587919.2018 .1520042 .

Leont'ev, Aleksei Nikolaevich. 1978. Activity, Consciousness, and Personality. Prentice-Hall Englewood Cliffs, NY. https://www.marxists.org/archive/leontev/works/activity-consciousness.pdf.

Linders, Dennis. 2012. «From E-Government to We-Government: Defining a Typology for Citizen Coproduction in the Age of Social Media». Government Information Quarterly 29 (4): 446-54. https://doi.org/10.1016/j.giq.2012.06.003.

Mayrberger, Kerstin, und Sandra Hofhues. 2013. «Akademische Lehre braucht mehr „Open Educational Practices“ für den Umgang mit „Open Educational Resources“ - ein Plädoyer.» Zeitschrift für Hochschulentwicklung - ZFHE 8(4): 56-66. https://doi.org/10.3217/zfhe8-04/07.

Mayrberger, Kerstin, und Stefan Thiemann. 2018. «Jenseits von Selbstreferenzialität - Awareness for Openness@UHH.»Synergie - Fachmagazin für Digitalisierung in der Lehre 5: 88-91. https://uhh.de/gxoal.

Mayrberger, Kerstin. (im Druck, 2019). «Lernendenorientierung im Kontext von Open Educational Practices.» In Digitalisierung und Lernen. Gestaltungsperspektiven für das professionelle Handeln in der Erwachsenenbildung und Weiterbildung, hrsg. v. Erik Haberzeth und Irena Sgier. Zürich: hep-Verlag.

Mayrberger, Kerstin. (in Vorbereitung). Partizipative Mediendidaktik. Weinheim: Beltz Juventa.

Mayrberger, Kerstin. 2014. «Partizipative Mediendidaktik. Inwiefern bedarf es im Kontext einer partizipativen Medienkultur einer spezifischen Mediendidaktik?» In Partizipative Medienkulturen. Positionen und Untersuchungen zu veränderten Formen öffentlicher Teilhabe, hrsg. v. Ralf Biermann, Johannes Fromme, Dan Verständig, 261-282. Wiesbaden: Springer VS. https://doi.org/10.1007/978-3-658-01793-4_12. 
Mayrberger, Kerstin. 2017. «Partizipatives Lernen in der Online-Lehre - Anspruch, Konzept und Ausblick». In Lehren und Lernen online. Lehr- und Lernerfahrungen im Kontext akademischer Online-Lehre, hrsg. v. Hedwig Rosa Griesehop und Edith Bauer, 109-129. Wiesbaden: Springer Fachmedien. https://doi.org/10.1007/978-3-658-15797-5_6.

Moon, Jennifer A. 2013. Reflection in Learning and Professional Development: Theory and Practice. 1. Aufl. London: Routledge. https://doi.org/10.4324/9780203822296.

Naidu, Som. 2016. Editorial. The case for open educational practice. Distance Education, 37 (1), 1-3. https://doi.org/10.1080/01587919.2016.1157010.

Neuhoff, Antje. 2012. "Shared Practices amongst Teachers in Online Training Courses.» Journal of e-Learning and Knowledge Society 8 (3): 105-118. https://doi.org/10.20368/19718829/646.

Okoli, Chitu, und Kira Schabram. 2010. «A guide to conducting a systematic literature review of Information Systems Research» Sprouts: Working Papers on Information Systems 10(26): 1-49. https://pdfs.semanticscholar.org/31dc/753345d5230e421ea817dd7dcdd352e87ea2. pdf.

OPAL - Open Educational Quality Initiative. 2011b. Beyond OER. Shifting Focus to Open Educational Practices in Organisations. OPAL-Report 2011. https://oerknowledgecloud.org/sites/ oerknowledgecloud.org/files/OPAL2011.pdf.

OPAL - Open Educational Quality Initiative. 2011a. OEP Guide. Guidelines for Open Educational Practices in Organisations. http://oerworkshop.pbworks.com/w/file/fetch/44605120/ OPAL-OEP-guidelines.pdf.

Peter, Julia. 2018. "Auf dem Weg zu inklusiver, chancengerechter und hochwertiger Bildung. Open Educational Resources aus UNESCO-Perspektive.» Synergie - Fachmagazin für Digitalisierung in der Lehre 5: 65-67. https://www.synergie.uni-hamburg.de/de/media/ausgabe05/synergie05-beitrag12-peter.pdf.

Petticrew, Mark, und Helen Roberts. 2006. Systematic Reviews in the Social Sciences: A Practical Guide. Oxford: Blackwell Publishing.

Reckwitz, Andreas. 2003. "Grundelemente einer Theorie sozialer Praktiken. Eine sozialtheoretische Perspektive.» Zeitschrift für Soziologie 32(4): 282-301. http://www.zfs-online.org/ index.php/zfs/article/view/1137/.

Reckwitz, Andreas. 2006. «Die historische Transformation der Medien und die Geschichte des Subjekts». In Medien der Gesellschaft - Gesellschaft der Medien, hrsg. v. Andreas Ziemann, 89-107. Konstanz: UVK.

Richter, Christoph, und Heidrun Allert. 2017. «Poetische Spielzüge als Bildungsoption in einer Kultur der Digitalität». In Digitalität und Selbst. Interdisziplinäre Perspektiven auf Subjektivierungs- und Bildungsprozesse, hrsg. v. Heidrun Allert, Michael Asmussen, und Christoph Richter. Bielefeld: transcript Verlag. https://doi.org/10.14361/9783839439456-011. 
Rost, M., Renzl, B., und J. Kaschube. 2014. "Organisationale Ambidextrie - Mit Kompetenzmodellen Mitarbeiter einbinden und Veränderung kommunizieren». In Kommunikation in Change und Risk, hrsg. v. M. Stumpf und S. Wehmeier, 33-55. Wiesbaden: Springer VS. https://doi.org/10.1007/978-3-658-00218-3_2.

Schön, Donald A. 1983. The Reflective Practitioner: How Professionals Think in Action. New York: Basic Books.

Schreurs, Bieke, Antoine Van den Beemt, Fleur Prinsen, Gabi Witthaus, Gráinne Conole, und Maarten De Laat. 2014. «An Investigation into Social Learning Activities by Practitioners in Open Educational Practices». International Review of Research in Open and Distance Learning 15 (4): 1-20. http://doi.org/10.19173/irrodl.v15i4.1905.

Stalder, Felix. 2018. «Herausforderungen der Digitalität jenseits der Technologie.» Synergie Fachmagazin für Digitalisierung in der Lehre 5: 8-15. https://www.synergie.uni-hamburg. de/de/media/ausgabe05/synergie05-beitrag01-stalder.pdf.

Vygotsky, Lev. 1978. Mind in society. The development of higher psychological processes. Herausgegeben von Michael Cole. Cambridge, MA, USA: Harvard University Press.

Wiley, David, und Hilton, John. 2018. Defining OER-Enabled Pedagogy». International Review of Research in Open and Distributed Learning 19 (4): 133-147. https://doi.org/10.19173/irrodl.v19i4.3601.

Wiley, David. 2017. «Defining the „Open“ in Open Content and Open Educational Ressources.» http://opencontent.org/definition/.

Zimmerman, Barry J. 2000. «Attaining Self-Regulation». In Handbook of Self-Regulation, herausgegeben von Monique Boekaerts, Paul R. Pintrich, und Moshe Zeidner, 13-39. San Diego: CA: Academic Press (Elsevier). https://doi.org/10.1016/B978-012109890-2/50031-7. 